\title{
Biomaterials for Enhancing Neuronal Repair
}

\begin{abstract}
Olivia V. Cangellaris ${ }^{1,2,3,4}$ and Martha U. Gillette ${ }^{1,3,4,5,6 *}$
${ }^{1}$ Department of Bioengineering, University of Illinois at Urbana-Champaign, Urbana, IL, United States, ${ }^{2}$ Medical Scholars Program, College of Medicine, University of Illinois at Urbana-Champaign, Urbana, IL, United States, ${ }^{3}$ Micro and Nanotechnology Laboratory, University of Illinois at Urbana-Champaign, Urbana, IL, United States, ${ }^{4}$ Beckman Institute for Advanced Science and Technology, University of Illinois at Urbana-Champaign, Urbana, IL, United States, ${ }^{5}$ Department of Cell and Developmental Biology, University of Illinois at Urbana-Champaign, Urbana, IL, United States, ${ }^{6}$ Neuroscience Program, University of Illinois at Urbana-Champaign, Urbana, IL, United States
\end{abstract}

As they differentiate from neuroblasts, nascent neurons become highly polarized and elongate. Neurons extend and elaborate fine and fragile cellular extensions that form circuits enabling long-distance communication and signal integration within the body. While other organ systems are developing, projections of differentiating neurons find paths to distant targets. Subsequent post-developmental neuronal damage is catastrophic because the cues for reinnervation are no longer active. Advances in biomaterials are enabling fabrication of micro-environments that encourage neuronal regrowth and restoration of function by recreating these developmental cues. This mini-review considers new materials that employ topographical, chemical, electrical, and/or mechanical cues for use in neuronal repair. Manipulating and integrating these elements in different combinations will generate new technologies to enhance neural repair.

Keywords: neuroregenerative therapy, neural scaffolds, topography, electrical stimulation, hydrogels, self-rolledup membranes, nerve-guide-conduits, flexible electronics

\section{INTRODUCTION}

Neurons are characterized by dendrites, multiple slender filamentous protrusions that receive and integrate incoming information, and a single axon, which transmits integrated signals downstream in a multicellular network. These cellular extensions are typically several times longer than the relatively small cell body and form a myriad of interconnections that enable humans to sense, integrate, remember, and respond to the world. Unlike other systems in the human body, cues for growth and repair in the nervous system are no longer active post-developmentally and, consequently, structural and functional losses following disease or damage are catastrophic. Neurological deficits contribute to over 600 classified neurological disorders and affect $\sim 50$ million people in the United States alone (Brown et al., 2005). Neurological disorders often result in debilitation rather than immediate death, and the personal and financial costs become staggering. The global burden of neurological afflictions, measured in disability-adjusted life years (DALYs), exceeds that of other diseases including heart disease and cancer (WHO, 2006). Therefore, new methods of treatment that ameliorate or resolve neurological disorders are necessary.

Innovative therapies for neurodegeneration and traumatic injury are emerging from novel biomaterials. Development of materials that support and nurture growth without introducing trauma while facilitating neural repair have the potential to alleviate peripheral neuropathies; diabetic sensory neuropathy or spinal cord trauma would benefit (Teng et al., 2002; Gu et al., 2014). New techniques and advances in material design, such as pore-enhanced hydrogels to promote 
neuronal alignment (Lee et al., 2015b), are facilitating targeted neuronal growth and repair. Innovations in neural monitoring through flexible, biodegradable electronics provide a means to understand these processes at a more fundamental level, as well as track and monitor repair in vivo (Viventi et al., 2011; Kang et al., 2016). These engineered interfaces address specific challenges inherent to damaged neural tissue by reducing glial scarring and overcoming limited distances of regeneration (Orive et al., 2009; Tam et al., 2014).

Unmodified planar substrates inadequately capitalize on endogenous factors that could enhance the efficacy of the substrate to promote targeted cellular development and growth. Modifying the substrate to better approximate the native developmental environment of neurons encourages the extension of neurites and repair of lesions. This review explores recent advances in the manipulation of topography, electric cues, and stiffness in biomaterials to enhance neuronal dynamics (e.g., neuritogenesis), improve growth, and allow monitoring of neural systems. Cues or properties are compared for relative impact on neuronal behavior and development (Table 1). While the integration of chemical cues into materials has been widely employed in other neuronal studies (Moore et al., 2006; Patel et al., 2007; Millet et al., 2010), the influence of chemical signals is intertwined in the discussion of the aforementioned parameters. This review focuses on neurons, while discussion of neural repair of all major cell populations within the nervous system, including glia, has been considered elsewhere (Schmidt and Leach, 2003; Tian et al., 2015).

\section{TOPOGRAPHICAL CUES DRIVE ALIGNMENT AND DIRECTIONALITY}

Cellular dynamics are strongly influenced by substrate topography (Bettinger et al., 2009; Ventre et al., 2012). Throughout the body, the extracellular matrix (ECM), with its fibers of collagen, fibronectin, and/or laminin, provides scaffolding that cells can adhere to and climb on, over, and through to travel to their terminal point. Neurons themselves can provide critical topography. An example is during formation of laminar brain structures, where new daughter cells use the scaffold provided by radial glial cells to migrate outward and form successive cortical layers (Rakic, 1972; Edmondson and Hatten, 1987; Kriegstein, 2005; Barros et al., 2011).

When designing customized materials and substrates for use in neural repair, the relationship between neuronal cells and native in vivo topography informs the relation to the desired functional outcome. Neuronal migration and neurite extension or directionality can be guided by the addition of topographical cues to a substrate, which enhances control by providing a

\footnotetext{
Abbreviations: DALYs, Disability-adjusted life years; ECM, Extracellular matrix; PLLA, Polylactic acid; DRG, Dorsal root ganglia; ESCs, Embryonic stem cells; iPSCs, Induced pluripotent stem cells; hBMSCs, Human bone marrow stromal cells; S-RuMs, Self-rolled-up membranes; NGCs, Nerve-guide-conduits or nerveguidance-channels; PHB-HV, Poly(3-hydroxybutyrate-co-3-hydroxyvalerate); EFs, Electric fields; DC, Direct current; AC, Alternating current; PPy, Polypyrrole; E, Elastic modulus; NPCs, Neural progenitor cells; CSF, Cerebral spinal fluid.
}

recognizable path (Jang et al., 2010; Baranes et al., 2012). In the case of damaged spinal neurons, a 3D scaffold can provide a sturdy framework to support directional neurite regrowth. A tubular design allows for directed tunneling of the neurite to the distal region needing reinnervation. Nanotopography is also important for cell adhesion and plays a critical role in material design (Yu et al., 2008; Khan and Newaz, 2010). Cellular adhesion depends on surface properties such as wettability and charge. These elements can be modified during fabrication and functionalization through protein deposition to the substrate surface (Subramanian et al., 2009). Furthermore, cells can respond to nanoscale features in ways that change morphology, attachment, proliferation, and even gene expression in response to nano-gratings, posts, and pits (Bettinger et al., 2009).

Polymer nanofibers are used to build scaffolds that support and direct neurite extension of neuron cultures in vitro. These scaffolds are fabricated using electrospinning, a technique that allows for accumulation of nanofibers in specific orientations. The process is highly customizable and the fibers can be spun in $\mathrm{nm}$ or $\mu \mathrm{m}$ scales (Pettikiriarachchi et al., 2010). Polylactic acid (PLLA) fibers of large diameter $(>1,000 \mathrm{~nm})$ have been shown to enhance neurite extension in dissociated chick dorsal root ganglia (DRG) cultures (Wang et al., 2010). Functionalizing PLLA fibers with fibronectin or laminin further improves neurite interaction by replicating these endogenous chemical cues (Koppes et al., 2014). Other electrospun nanofiber scaffolds improve DRG neurite extension, promote differentiation of mouse embryonic stem cells (ESCs) into neural progenitors, and enhance outgrowth of neurites on the scaffolds with aligned fibers. Neural crest stem cells differentiated from iPSCs cultured within nanofiber-modified conduits enhanced sciatic nerve regeneration (Xie et al., 2009; Schaub and Gilbert, 2011; Wong et al., 2011). Nanofibers can be spun from a variety of biocompatible materials, including natural proteins such as collagen. However, there are several limitations to these scaffolds. It is difficult to create an environment mimicking the endogenous ECM, because its components are smaller than what is currently achievable when fabricating nanofibers $(\sim 100 \mathrm{~nm}$ thick). Additionally, nanofiber scaffolds cannot support embedded cells without compromising the structural integrity of the scaffold (Liu et al., 2012).

Hydrogels, networks of polymers that have been swollen with water, are attractive materials for cellular applications due to their biocompatibility, ease of fabrication, and capacity for customization (Caliari and Burdick, 2016). One advantage of hydrogels is that their porosity is not detrimental to their structure and can allow for migration of cells within the hydrogel scaffold. Hydrogels fabricated with an additional internal topography promote alignment or directionality of hippocampal and DRG neurons (Liu et al., 2015), and differentiation of stem cells into a neuronal cell-type (Lee et al., 2015b). When human bone marrow stromal cells (hBMSCs) were cultured in hydrogels with both aligned microchannels (Figure 1A) and stochastically formed micropores (Figure 1B), hBMSCs differentiated into neuronal cells and elongated to grow within the microchannels (Figure 1C). Differentiation was attributed to the topography facilitating binding between cellular integrins and ligands, which 
TABLE 1 | Impact of various material properties on neuronal behavior and development.

\begin{tabular}{|c|c|c|c|}
\hline Material property & Neurites & Directionality & Cell fate \\
\hline Topographical $^{\mathrm{a}}$ & - Increased neurite length & $\begin{array}{l}\text { Neurite direction guided by } \\
\text { - Tubular structures } \\
\text { - Microchannels } \\
\text { - Confined spaces }\end{array}$ & $\begin{array}{l}\text { iPSCs, ESCs, hBMSCs differentiate to } \\
\text { neural cell type }\end{array}$ \\
\hline Electrical $^{\mathrm{b}}$ & $\begin{array}{l}\text { - Increased neurite length } \\
\text { - Enhanced neuritogenesis }\end{array}$ & $\begin{array}{l}\text { - Neurites grow/extend in direction of EF } \\
\text { - Neurite growth rate increased } \\
\text { - Neurons migrate in EF direction } \\
\text { - Polarization of neurons }\end{array}$ & $\begin{array}{l}\text { - Direct neural tube formation } \\
\text { - Direct cell migration and organization } \\
\text { - Influence neuronal differentiation }\end{array}$ \\
\hline Mechanical Stiffness ${ }^{C}$ & $\begin{array}{l}\text { Decreased stiffness supports increased neurite length } \\
\text { Increased stiffness results in: } \\
\text { - Improved network connectivity } \\
\text { - Improved signal transduction }\end{array}$ & No effect & $\begin{array}{l}\text { Decreased stiffness directs stem cell } \\
\text { differentiation toward the neural lineage }\end{array}$ \\
\hline
\end{tabular}

aTopographical References: (Xie et al., 2009; Schaub and Gilbert, 2011; Wong et al., 2011; Froeter et al., 2014; Koppes et al., 2014; Lee et al., 2015b).

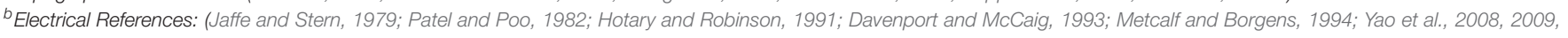
2011; Graves et al., 2011; Koppes et al., 2014; Kim et al., 2016; Ma et al., 2016).

cMechanical Stiffness References: (Balgude et al., 2001; Discher et al., 2005; Jiang et al., 2010; Keung et al., 2012; Lee et al., 2013; Zhang et al., 2014; Mosley et al., 2017).

is important for stem cell differentiation to neurons. The stochastic micropore gels could not support this binding, leading to mostly undifferentiated hBMSCs (Lee et al., 2015b). Hydrogels can also be used for cell encapsulation or fabricated with particles bearing trophic factors to enhance cellular interactions on and within the gel (Carballo-Molina and Velasco, 2015).

A semiconductor-based microtube substrate, composed of a thin nanomembrane of oppositely strained layers of silicon nitride that can self-roll, significantly enhances neurite alignment (Figure 1D). These self-rolled-up membranes (S-RuMs) have a unique combination of features that make them attractive for manipulating topography. S-RuMs are optically transparent under most conventional microscopy techniques, including phase-contrast and fluorescence imaging, which makes them ideal for use with cultured cells. Since they are manufactured using a scalable semiconductor process (Li, 2008; Huang et al., 2012), they are highly customizable and versatile, which facilitates many different designs (Froeter et al., 2013). They also are biocompatible, an essential characteristic for cell and tissue interfaces (Froeter et al., 2014). The S-RuMs can be tuned to a range of diameters and lengths, can be rolled into a single or binocular tube, and can be incorporated with pores to allow for nutrient and gas exchange across the tube membrane. By restricting the diameter of the $\mathrm{S}-\mathrm{RuM}$ to the $5-\mu \mathrm{m}$ range, a single neurite can be captured within each tube. By altering the fabrication process to widen the diameter, a bundle of neurites can traverse a single tube. Additionally, a thin deposition of metal can be added during the fabrication process to create an electrode that is rolled within the S-RuM (Figure 1E). This characteristic will enable selective and targeted stimulation and recording of a neurite contained on a single substrate and continuous tracking of functional neurite dynamics under electrical stimulation. Scanning electron microscopy (SEM) of rat hippocampal neurons in culture reveal the S-RuMs provide adequate space for neurites to extend, turn, and extend through the lumen (Figure 1F).
Nerve-guide-conduits or nerve-guidance-channels (NGCs) are $3 \mathrm{D}$ constructs for whole nerve therapies in vivo (Anderson et al., 2017; Lackington et al., 2017). They are currently used as implants for neural repair in humans. Commercially available NGCs are primarily single-lumen tubes, with no added topographical features, through which the two ends of a severed nerve are inserted and left to grow together (de Ruiter et al., 2009). There are limitations to these models, most notably in the injury gap distance over which they are effective. Functionalization to improve rate of regrowth, limit scarring, and improve permeability for nutrient transfer has yet to be integrated into these devices.

Techniques that have proven successful during in vitro neuroregenerative studies are currently being applied and evaluated in NGCs in animal models. An experimental NGC, composed of poly(3-hydroxybutyrate-co-3-hydroxyvalerate) (PHB-HV) and enhanced with a conductive polypyrrole co-polymer coating along the inner diameter of the NGC, has been implanted in Sprague-Dawley rats with severed sciatic nerves. When the conduits were harvested at 8 weeks and analyzed for neuronal markers, nerve tissue was found throughout the conduit with no evidence of inflammation. Thus, the NGC supports and promotes regeneration of damaged nerves (Durgam et al., 2010). A more recent study in rats demonstrated nerve regeneration in vivo that utilized NGCs made of zein, a corn-derived polymer. NGCs were fabricated in three configurations: non-porous NGCs, porous NGCs, and porous NGCs that contained smaller zein microtubes. A $10-\mathrm{mm}$ section of the sciatic nerve was removed and replaced with the NGCs, and recovery was tracked over a 4-month period. The rats showed improved gait 2 months after implantation. The porous zein conduit showed significantly increased density of myelinated nerve fibers and increased myelin sheath thickness at 2- and 4-months post-implantation (Wang et al., 2017). The porous nature of these zein NGCs enabled nutrient diffusion and facilitated eventual degradation of the scaffold over the 

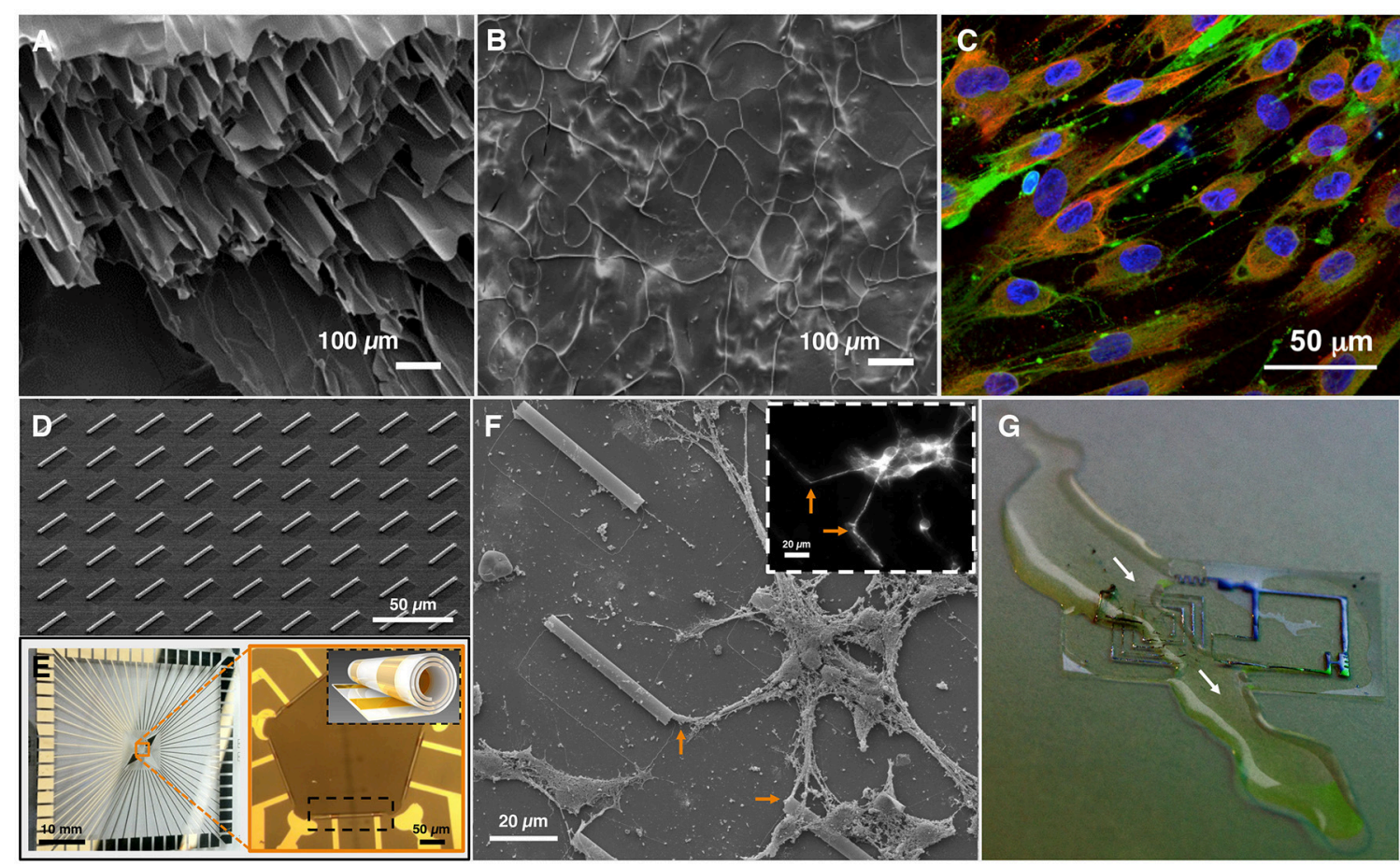

G

FIGURE 1 | Material applications of topography, electrical stimulation, and stiffness. (A) Scanning electron micrograph (SEM) of fractured hydrogels reveals the internal structure of the hydrogel with microchannels, or (B) micropores (images A,B adapted from Lee et al., 2015a). (C) Human bone marrow stromal cells (hBMSCs) cultured on hydrogel with aligned microchannels differentiate into a neuronal phenotype. Fluorescence imaging reveals MAP2 (neuronal marker, green), GFAP (glial marker, red), and DAPI (nuclear marker, blue) immunoreactivity, demonstrating differentiation of hBMSCs into cells expressing neuronal or glial markers, and elongating in the microchanneled hydrogel (image contributed by $\mathrm{H}$. J. Kong, University of Illinois at Urbana-Champaign). (D) SEM of array of self-rolled-up membranes (S-RuMs) composed of thin-film silicon nitride bilayers. (E) A multi-electrode array chip (left) with S-RuMs patterned in a pentagon formation (orange box, right). Black inset shows schematic of single S-RuM with gold electrodes rolled inside (images D,E contributed by X. Li, University of Illinois at Urbana-Champaign). (F) SEM of rat hippocampal neurons cultured on S-RuM substrate (3 days in vitro). Inset: Fluorescence imaging reveals MAP2 (neuronal marker, white) immunoreactivity, confirming neuronal cell type. Orange arrows correspond to entry of neurites into S-RuMs. (G) An example of flexible, biocompatible, dissolvable electronics: an electronic circuit dissolving in a stream of water. White arrows indicate the path of the water and the region of the circuit that is dissolving (image contributed by J.A. Rogers, Northwestern University).

course of 4 months, when nerve regeneration in the conduit with microtubes was comparable to the regeneration observed in autograft controls (Wang et al., 2017). Collectively, these results highlight how topography can positively promote neurite outgrowth and enhance regeneration.

\section{APPLICATION OF ELECTRIC FIELDS TO MANIPULATE NEURITE EXTENSION}

The nervous system relies on electrical signals for development and communication. In early development, electric potentials define migration paths of the cells and differentiation, driving the formation of the neural tube (Hotary and Robinson, 1990, 1991; Metcalf and Borgens, 1994; Yao et al., 2008; Ma et al., 2016). Signal transmission in neurons is mediated by ion fluxes across the cell membrane. In instances of traumatic injury, ion flux establishes an electric potential gradient that promotes repair (Reid et al., 2007; McCaig et al., 2009). Numerous studies support the positive effect of electric fields (EFs) on neurite extension, growth-rate, and neuron polarization and migration (Jaffe and Stern, 1979; Patel and Poo, 1982; McCaig, 1990; Davenport and McCaig, 1993; Yao et al., 2009, 2011; Graves et al., 2011; Kim et al., 2016). Consequently, electrical cues can be utilized to positively regulate, facilitate, and enhance neuroregeneration.

Nanofiber scaffolds can be augmented to enhance neurite outgrowth by providing both electrical stimulation and topographical cues. An external EF was introduced in parallel or perpendicular orientation to planar PLLA fiber scaffolds using an agar salt-bridge and platinum reference electrode. When rat DRG cultured on each of these scaffolds were stimulated with an applied direct current (DC) EF for $8 \mathrm{~h}$, neurite outgrowths on the electrically stimulated scaffolds were significantly longer compared to controls. Neurite outgrowth increased by $74 \%$ on the PLLA fibers (topography alone), 32\% on the PLLA planar films (electrical stimulation alone), and by $126 \%$ on the PLLA fibers aligned to the direction of the DC EF (Koppes et al., 2014). Therefore, the combination of topographical and electrical cues greatly improves length of neurite extension. Electrical stimulation can be further integrated into the scaffold 
by choosing a conductive base material. Polypyrrole (PPy) is biocompatible, biodegradable, as well as electrically conductive, so NGCs augmented with PPy can support electrical stimulation (Nguyen et al., 2014). When DRGs on PPy-modified flat scaffolds were stimulated with an electric field, neurite extension was enhanced by $13 \%$ in a DC EF, and $21 \%$ in an alternating current (AC) EF. PPy-modified NGCs without electrical stimulation were shown to support regrowth of severed sciatic nerves in rats in vivo (Durgam et al., 2010). To translate this technology to in vivo models, electrical stimulation needs to be introduced to a PPy-modified NGC. Electric stimulation is a native signal that strongly impacts neurons and can be further manipulated to direct neuritogenesis in strategies for neuroregenerative therapy.

\section{MANIPULATION OF SUBSTRATE STIFFNESS}

The intrinsic mechanical properties of the body determine neuronal differentiation, dynamics, behavior, and organization (Hynes, 2009; Janmey and Miller, 2011; Koser et al., 2016). The importance of substrate mechanics as a cue is evident during differentiation of stem cells in environments of controlled stiffness. Increasingly higher stiffness encourages their differentiation into muscle [elastic modulus (E) $\sim 10 \mathrm{kPa}$ ] or bone $(E>30 \mathrm{kPa})$, whereas a lower stiffness on the order of hundreds of $\mathrm{Pa}$ encourages differentiation into neurons (Lee et al., 2013). This is consistent with the elastic modulus within the central and peripheral nervous systems, which ranges between 0.5 and $1 \mathrm{kPa}$, and the shear stiffness of human brain tissue in vivo, which has been measured between 2 and $3 \mathrm{kPa}$ (Lee et al., 2013; Bai et al., 2014; Hiscox et al., 2016). Such measurements of human brain tissue are highly dependent on frequency and region, and therefore variable. Additional studies have demonstrated that timing and duration of exposure to stiffness cues impacts stem cell differentiation to neural cell types, and that while neuritogenesis may be enhanced on soft substrates, network connectivity and signal transduction are enhanced by stiffer substrates (Balgude et al., 2001; Jiang et al., 2010; Keung et al., 2012; Zhang et al., 2014; Mosley et al., 2017). These findings emphasize that stiffness cues should be adjusted depending on the desired outcome, with close attention to the region of interest in the human body.

Hydrogels are a compelling choice for neuronal scaffolds because their elastic modulus is easily tuned during fabrication, although dependent upon the monomer/material used. Polyacrylamide can be used to create hydrogels with gradient stiffness ranging from $\sim 1$ to $240 \mathrm{kPa}$. Polyacrylamide can act as a strong analog to the endogenous ECM when invested with proteins and chemical signals specific to the cell of interest (Sunyer et al., 2012; Lee et al., 2015b). Hydrogels can be constructed in planar or $3 \mathrm{D}$ configurations maintaining precise control over the elastic modulus (Chatterjee et al., 2011; Wylie et al., 2011). They facilitate nutrient exchange and diffusion of gasses through their natural pores. This exchange contributes to healthier cells within the deepest parts of the scaffold. In designing scaffolds for use in repair of nervous tissue, manipulating the base material to more closely resemble the endogenous elastic modulus can facilitate more natural integration with the existing cellular structure.

\section{INNOVATIVE SUBSTRATES FOR EFFECTIVE REPAIR}

An ideal substrate for effective repair should take into account a combination of topographical, chemical, electrical, and mechanical properties of the substrate. The parameters must be carefully tailored to address the site of application, as biocompatibility with surrounding tissue will differ, and the time course for repair, which will influence the duration of the implant. For an acute spinal cord injury, the ideal substrate should facilitate the initial regrowth, and protect against glial scarring while nurturing the damaged axons during the healing process via embedded trophic factors. Once the lesion has healed and the scaffold has served its purpose, the scaffold can either be resorbed or fully integrated into the recovered tissue. Such a substrate must be flexible with an elastic modulus matching the native spinal column for an environment that closely resembles the endogenous condition. The scaffold can be enriched with microchannels, which attract the regenerating neurites given their affinity for edges and enclosed spaces (Millet et al., 2007; Froeter et al., 2014; Li et al., 2015). To enhance regrowth and influence its directionality, electronics that support electrical stimuli can be embedded in the scaffold. These electronics can also support recording capabilities to assess neuronal activity. Impregnating the scaffold with stem cells could enhance this therapy even more.

A recent study demonstrated how grafted human spinal cordderived neural progenitor cells (NPCs) restore functionality to primates with lesioned spinal cords. The NPCs survived in the graft 9 months following injury and enabled recovered functionality in the primate forelimbs. Two notable challenges were encountered before a successful grafting method was developed: (1) in initial grafts, the NPCs were washed away by the native cerebral spinal fluid (CSF) that refilled the lesion site, and (2) the initial immunosuppressive regimen was not robust enough to enable the graft to survive the host immune response, leading to poor filling of the lesion with the NPCs. These two challenges were resolved by draining the CSF in the region of the lesion prior to grafting, increasing the grafting mixture to hasten the rate of gelling, subjecting the primates to higher initial doses of the immunosuppressants, and monitoring the subjects more frequently (Rosenzweig et al., 2018). The success of this study could be improved by loading the NPCs on an idealized scaffold as described above, which would protect the NPCs and allow for active monitoring of the regeneration.

With advancements in materials engineering, a new wave of flexible and biodegradable electronics has been introduced (Figure 1G). Applications for their use in the nervous system are especially promising. Flexible, transient, silicon-based, biocompatible, implantable biosensors are being developed that allow for wireless monitoring capability. They have been used successfully on skin, cardiac tissue, muscle, and the brain (Viventi et al., 2010, 2011; Hwang et al., 2012; Kang et al., 2016). A wireless communication device composed of bioresorbable 
materials has been successfully implanted and used in rats for monitoring intracranial pressure and temperature (Kang et al., 2016). Another flexible, non-penetrating multi-electrode array with embedded ultrathin silicon transistors was used for in vivo neural recording and monitoring of electrical brain activity in feline models. The electrode array was applied to the visual cortex, or folded and inserted into the interhemispheric fissure, and electrical signals corresponding to visual stimuli were recorded (Viventi et al., 2011). The connection between these technologies and solutions for neuronal repair lies in three major advantages of these devices: (1) the flexible nature of the material allows for intimate contact between the biosensor and the neural tissue, minimizing current-loss (Viventi et al., 2010, 2011), (2) the materials are biocompatible and do not trigger an inflammatory response (Kang et al., 2016), and (3) the devices are bioresorbable. Each of these elements addresses requirements of an ideal substrate for neural repair. While long-term clinical translation of these devices must ensure longevity of the materials and sustained biocompatibility, progress in flexible electronics development is promising.

\section{CONCLUSION}

Recovering function following damage to neuronal systems is challenging due to loss of native cues, inflammation, and scarring. Solutions to this problem lie in clever development and functionalization of new scaffolds on which neurons can regenerate complex, 3D circuits. Important advances are being made in development of biomaterials for neuronal repair, including: (1) the development of new polymer/co-polymer substrates to enhance scaffolds for better integration with neural tissue, (2) new topographical structures to heighten neurite capture, support, and growth, and (3) novel manipulations of silicon-based electronics to design and implement flexible substrates for stimulation and recording. New materials enabling manipulation of substrate topography, such as hydrogels, mimic similar in vivo structures and enhance control over directionality

\section{REFERENCES}

Anderson, M., Shelke, N. B., Manoukian, O. S., Yu, X., McCullough, L. D., and Kumbar, S. G. (2017). Peripheral nerve regeneration strategies: electrically stimulating polymer based nerve growth conduits. Crit. Rev. Biomed. Eng. 43, 131-159. doi: 10.1615/CritRevBiomedEng.2015014015

Bai, S., Zhang, W., Lu, Q., Ma, Q., Kaplan, D. L., and Zhu, H. (2014). Silk nanofiber hydrogels with tunable modulus to regulate nerve stem cell fate. J. Mater. Chem. B. Mater. Biol. Med. 2, 6590-6600. doi: 10.1039/C4TB0 0878B

Balgude, A. P., Yu, X., Szymanski, A., and Bellamkonda, R. V. (2001). Agarose gel stiffness determines rate of DRG neurite extension in 3D cultures. Biomaterials 22, 1077-1084. doi: 10.1016/S0142-9612(00)00350-1

Baranes, K., Chejanovsky, N., Alon, N., Sharoni, A., and Shefi, O. (2012). Topographic cues of nano-scale height direct neuronal growth pattern. Biotechnol. Bioeng. 109, 1791-1797. doi: 10.1002/bit. 24444

Barros, C. S., Franco, S. J., and Müller, U. (2011). Extracellular matrix: functions in the nervous system. Cold Spring Harb. Perspect. Biol. 3:a005108. doi: $10.1101 /$ cshperspect.a005108 in regenerating neurites. Introduction of electrical stimulation will amplify growth rate and length of regeneration, and influence orientation. Embedded wireless sensors will enable real-time monitoring of regenerating nerves in situ. Substrates can be manipulated to further emulate the endogenous neural environment by tuning the elastic modulus to better match the range of local stiffnesses in vivo and provide transitions between native tissue and supportive scaffold. By developing scaffolds and devices that dissolve away after fulfilling their purpose, the need for an additional surgery for removal is eliminated, thereby reducing the risks of added surgical complications, such as infection, as well as additional medical costs. For the brain and the nervous system, the future is pliable and electronic.

\section{AUTHOR CONTRIBUTIONS}

The manuscript was conceived and prepared by OVC. OVC and MUG revised the manuscript.

\section{FUNDING}

The authors acknowledge funding from the Medical Scholars Program at the University of Illinois and Christie Foundation Award (OVC), and the National Science Foundation STC Emergent Behaviors of Integrated Cellular Systems CBET 0939511 (MUG).

\section{ACKNOWLEDGMENTS}

The authors thank Guillermo L. Monroy for contributions to revising the manuscript, Jennifer W. Mitchell for insights in figure preparation, and Ann C. Benefiel for facilitating manuscript submission. The authors thank Xiuling Li, Paul Froeter, and Hyun Joon Kong (University of Illinois at UrbanaChampaign), and John A. Rogers (Northwestern University) for providing images.
Bettinger, C. J., Langer, R., and Borenstein, J. T. (2009). Engineering substrate topography at the micro- and nanoscale to control cell function. Angew. Chemie Int. Ed. 48, 5406-5415. doi: 10.1002/anie.200805179

Brown, R. C., Lockwood, A. H., and Sonawane, B. R. (2005). Neurodegenerative diseases: an overview of environmental risk factors. Environ. Health Perspect. 113, 1250-1256. doi: 10.1289/ehp.7567

Caliari, S. R., and Burdick, J. A. (2016). A practical guide to hydrogels for cell culture. Nat. Methods 13, 405-414. doi: 10.1038/nmeth.3839

Carballo-Molina, O. A., and Velasco, I. (2015). Hydrogels as scaffolds and delivery systems to enhance axonal regeneration after injuries. Front. Cell. Neurosci. 9:13. doi: 10.3389/fncel.2015.00013

Chatterjee, K., Young, M. F., and Simon, C. G. Jr. (2011). Fabricating gradient hydrogel scaffolds for 3D cell culture. Comb. Chem. High Throughput Screen. 14, 227-236. doi: 10.2174/138620711795222455

Davenport, R. W., and McCaig, C. D. (1993). Hippocampal growth cone responses to focally applied electric fields. J. Neurobiol. 24, 89-100. doi: 10.1002/neu.480240108

de Ruiter, G. C., Malessy, M. J., Yaszemski, M. J., Winderbank, A. J., and Spinner, R. J. (2009). Designing ideal conduits for peripheral nerve repair. Neurosurg. Focus 26:E5. doi: 10.3171/FOC.2009.26.2.E5 
Discher, D., Janmey, P., and Wang, Y. (2005). Tissue cells feel and respond to the stiffness of their substrate. Science 310, 1139-1143. doi: 10.1126/science.1116995

Durgam, H., Sapp, S., Deister, C., Khaing, Z., Chang, E., Luebben, S., et al. (2010). Novel degradable co-polymers of polypyrrole support cell proliferation and enhance neurite outgrowth with electrical stimulation. J. Biomater. Sci. Polym. Ed. 21, 1265-1282. doi: 10.1163/092050609X12481751806330

Edmondson, J. C., and Hatten, M. E. (1987). Glial-guided granule neuron migration in vitro: a high-resolution time-lapse video microscopic study. J. Neurosci. 7, 1928-1934.

Froeter, P., Huang, Y., Cangellaris, O. V., Huang, W., Dent, E. W., Gillette, M. U., et al. (2014). Toward intelligent synthetic neural circuits: directing and accelerating neuron cell growth by self-rolled-up silicon nitride microtube array. ACS Nano 8, 11108-11117. doi: 10.1021/nn504876y

Froeter, P., Yu, X., Huang, W., Du, F., Li, M., Chun, I., et al. (2013). 3D hierarchical architectures based on self-rolled-up silicon nitride membranes. Nanotechnology 24:475301. doi: 10.1088/0957-4484/24/47/475301

Graves, M. S., Hassell, T., Beier, B. L., Albors, G. O., and Irazoqui, P. P. (2011). Electrically mediated neuronal guidance with applied alternating current electric fields. Ann. Biomed. Eng. 39, 1759-1767. doi: 10.1007/s10439-011-0259-8

Gu, X., Ding, F., and Williams, D. F. (2014). Neural tissue engineering options for peripheral nerve regeneration. Biomaterials 35, 6143-6156. doi: 10.1016/j.biomaterials.2014.04.064

Hiscox, L. V., Johnson, C. L., Barnhill, E., McGarry, M. D., Huston, J., van Beek, E. J., et al. (2016). Magnetic resonance elastography (MRE) of the human brain: technique, findings and clinical applications. Phys. Med. Biol. 61, R401-R437. doi: 10.1088/0031-9155/61/24/R401

Hotary, K. B., and Robinson, K. R. (1990). Endogenous electrical currents and the resultant voltage gradients in the chick embryo. Dev. Biol. 140, 149-160. doi: 10.1016/0012-1606(90)90062-N

Hotary, K. B., and Robinson, K. R. (1991). The neural tube of the Xenopus embryo maintains a potential difference across itself. Dev. Brain Res. 59, 65-73. doi: 10.1016/0165-3806(91)90030-M

Huang, W., Yu, X., Froeter, P., Xu, R., Ferreira, P., and Li, X. (2012). On-chip inductors with self-rolled-up SiNx nanomembrane tubes: a novel design platform for extreme miniaturization. Nano Lett. 12, 6283-6288. doi: $10.1021 / \mathrm{nl} 303395 \mathrm{~d}$

Hwang, S. W., Tao, H., Kim, D.-H., Cheng, H., Song, J.-K., Rill, E., et al. (2012). A physically transient form of silicon electronics, with integrated sensors, actuators and power supply. Science 337, 1640-1644. doi: $10.1126 /$ science. 1226325

Hynes, R. O. (2009). Extracellular matrix: not just pretty fibrils. Science 326, 1216-1219. doi: 10.1126/science.1176009

Jaffe, L. F., and Stern, C. D. (1979). Strong electrical currents leave the primitive streak of chick embryos. Science 206, 569-571. doi: 10.1126/science.573921

Jang, K. J., Kim, M. S., Feltrin, D., Jeon, N. L., Suh, K. Y., and Pertz, O. (2010). Two distinct filopodia populations at the growth cone allow to sense nanotopographical extracellular matrix cues to guide neurite outgrowth. PLoS ONE 5:e15966. doi: 10.1371/journal.pone.0015966

Janmey, P. A., and Miller, R. T. (2011). Mechanisms of mechanical signaling in development and disease. J. Cell Sci. 124, 9-18. doi: 10.1242/jcs.071001

Jiang, F. X., Yurke, B., Schloss, R. S., Firestein, B. L., and Langrana, N. A. (2010). Effect of dynamic stiffness of the substrates on neurite outgrowth by using a DNA-crosslinked hydrogel. Tissue Eng. A 16, 1873-1889. doi: 10.1089/ten.tea.2009.0574

Kang, S. K., Murphy, R. K., Hwang, S. W., Lee, S. M., Harburg, D. V., Krueger, N. A., et al. (2016). Bioresorbable silicon electronic sensors for the brain. Nature 530, 71-76. doi: 10.1038/nature 16492

Keung, A. J., Asuri, P., Kumar, S., and Schaffer, D. V. (2012). Soft microenvironments promote the early neurogenic differentiation but not self-renewal of human pluripotent stem cells. Integr. Biol. 4, 1049-1058. doi: $10.1039 / \mathrm{c} 2 \mathrm{ib} 20083 \mathrm{j}$

Khan, S., and Newaz, G. (2010). A comprehensive review of surface modification for neural cell adhesion and patterning. J. Biomed. Mater. Res. Part A 93, 1209-1224. doi: 10.1002/jbm.a.32698

Kim, K. M., Kim, S. Y., and Palmore, G. T. (2016). Axon outgrowth of rat embryonic hippocampal neurons in the presence of an electric field. ACS Chem. Neurosci. 7, 1325-1330. doi: 10.1021/acschemneuro. $6 \mathrm{~b} 00191$

Koppes, A. N., Zaccor, N. W., Rivet, C. J., Williams, L. A., Piselli, J. M., Gilbert, R. J., et al. (2014). Neurite outgrowth on electrospun PLLA fibers is enhanced by exogenous electrical stimulation. J. Neural Eng. 11:46002. doi: 10.1088/1741-2560/11/4/046002

Koser, D. E., Thompson, A. J., Foster, S. K., Dwivedy, A., Pillai, E. K., Sheridan, G. K., et al. (2016). Mechanosensing is critical for axon growth in the developing brain. Nat. Neurosci. 19, 1592-1598. doi: 10.1038/nn.4394

Kriegstein, A. R. (2005). Constructing circuits: neurogenesis and migration in the developing neocortex. Epilepsia 46, 15-21. doi: 10.1111/j.1528-1167.2005.00304.x

Lackington, W. A., Ryan, A. J., and O’Brien, F. J. (2017). Advances in nerve guidance conduit-based therapeutics for peripheral nerve repair. ACS Biomater. Sci. Eng. 3, 1221-1235. doi: 10.1021/acsbiomaterials.6b00500

Lee, J., Abdeen, A. A., Zhang, D., and Kilian, K. A. (2013). Directing stem cell fate on hydrogel substrates by controlling cell geometry, matrix mechanics and adhesion ligand composition. Biomaterials 34, 8140-8148. doi: 10.1016/j.biomaterials.2013.07.074

Lee, M. K., Rich, M. H., Baek, K., Lee, J., and Kong, H. (2015a). Bioinspired tuning of hydrogel permeability-rigidity dependency for 3D cell culture. Sci. Rep. 5:8948. doi: 10.1038/srep0894

Lee, M. K., Rich, M. H., Lee, J., and Kong, H. (2015b). A bio-inspired, microchanneled hydrogel with controlled spacing of cell adhesion ligands regulates $3 \mathrm{D}$ spatial organization of cells and tissue. Biomaterials 58, 26-34. doi: 10.1016/j.biomaterials.2015.04.014

Li, W., Tang, Q. Y., Jadhav, A. D., Narang, A., Qian, W. X., Shi, P., et al. (2015). Large-scale topographical screen for investigation of physical neural-guidance cues. Sci. Rep. 5:8644. doi: 10.1038/srep08644

Li, X. (2008). Strain induced semiconductor nanotubes: from formation process to device applications. J. Phys. D. Appl. Phys. 41:193001. doi: 10.1088/0022-3727/41/19/193001

Liu, S. C., Lee, M. K., Slater, B. J., Kouzehgarani, G. N., Yu, M., Cangellaris, O. V., et al. (2015). "Engineering a 3D platform to mimic in vivo neural network morphology and activity," in Program No. P030. 2015 Neuroscience Meeting Planner (Chicago, IL: Society for Neuroscience).

Liu, W., Thomopoulos, S., and Xia, Y. (2012). Electrospun nanofibers for regenerative medicine. Adv. Healthc. Mater. 1, 10-25. doi: 10.1002/adhm.201100021

Ma, Q., Chen, C., Deng, P., Zhu, G., Lin, M., Zhang, L., et al. (2016). Extremely lowfrequency electromagnetic fields promote in vitro neuronal differentiation and neurite outgrowth of embryonic neural stem cells via up-regulating TRPC1. PLoS ONE 11:e0150923. doi: 10.1371/journal.pone.0150923

McCaig, C. D. (1990). Nerve branching is induced and oriented by a small applied electric field. J. Cell Sci. 95(Pt 4), 605-615.

McCaig, C. D., Song, B., and Rajnicek, A. M. (2009). Electrical dimensions in cell science. J. Cell Sci. 122, 4267-4276. doi: 10.1242/jcs.023564

Metcalf, M. E. M., and Borgens, R. B. (1994). Weak applied voltages interfere with amphibian morphogenesis and pattern. J. Exp. Zool. 268, 323-338. doi: 10.1002/jez.1402680408

Millet, L. J., Stewart, M. E., Nuzzo, R. G., and Gillette, M. U. (2010). Guiding neuron development with planar surface gradients of substrate cues deposited using microfluidic devices. Lab Chip 10, 1525-1535. doi: 10.1039/c001552k

Millet, L. J., Stewart, M. E., Sweedler, J. V., Nuzzo, R. G., and Gillette, M. U. (2007). Microfluidic devices for culturing primary mammalian neurons at low densities. Lab Chip 7, 987-994. doi: 10.1039/b705266a

Moore, K., Macsween, M., and Shoichet, M. (2006). Immobilized concentration gradients of neurotrophic factors guide neurite outgrowth of primary neurons in macroporous scaffolds. Tissue Eng. 12, 267-278. doi: 10.1089/ten.2006.12.267

Mosley, M. C., Lim, H. J., Chen, J., Yang, Y. H., Li, S., Liu, Y., et al. (2017). Neurite extension and neuronal differentiation of human induced pluripotent stem cell derived neural stem cells on polyethylene glycol hydrogels containing a continuous Young's Modulus gradient. J. Biomed. Mater. Res. Part A 105, 824-833. doi: 10.1002/jbm.a.35955

Nguyen, H. T., Sapp, S., Wei, C., Chow, J. K., Nguyen, A., Coursen, J., et al. (2014). Electric field stimulation through a biodegradable polypyrrole-copolycaprolactone substrate enhances neural cell growth. J. Biomed. Mater. Res. Part A 102, 2554-2564. doi: 10.1002/jbm.a.34925 
Orive, G., Anitua, E., Pedraz, J. L., and Emerich, D. F. (2009). Biomaterials for promoting brain protection, repair and regeneration. Nat. Rev. Neurosci. 10, 682-692. doi: $10.1038 / \mathrm{nrn} 2685$

Patel, N., and Poo, M. M. (1982). Orientation of neurite growth by extracellular electric fields. J. Neurosci. 2, 483-496.

Patel, S., Kurpinski, K., Quigley, R., Gao, H., Hsiao, B. S., Poo, M. M., et al. (2007). Bioactive nanofibers: synergistic effects of nanotopography and chemical signaling on cell guidance. Nano Lett. 7, 2122-2128. doi: 10.1021/nl071182z

Pettikiriarachchi, J. T. S., Parish, C. L., Shoichet, M. S., Forsythe, J. S., and Nisbet, D. R. (2010). Biomaterials for brain tissue engineering. Aust. J. Chem. 63, 1143-1154. doi: 10.1071/CH10159

Rakic, P. (1972). Mode of cell migration to the superficial layers of fetal monkey neocortex. J. Comp. Neurol. 145, 61-83. doi: 10.1002/cne.901450105

Reid, B., Nuccitelli, R., and Zhao, M. (2007). Non-invasive measurement of bioelectric currents with a vibrating probe. Nat. Protoc. 2, 661-669. doi: $10.1038 /$ nprot.2007.91

Rosenzweig, E. S., Brock, J. H., Lu, P., Kumamaru, H., Salegio, E. A., Kadoya, K., et al. (2018). Restorative effects of human neural stem cell grafts on the primate spinal cord. Nat. Med. doi: 10.1038/nm.4502. [Epub ahead of print].

Schaub, N. J., and Gilbert, R. J. (2011). Controlled release of 6-aminonicotinamide from aligned, electrospun fibers alters astrocyte metabolism and dorsal root ganglia neurite outgrowth. J. Neural Eng. 8:046026. doi: 10.1088/1741-2560/8/4/046026

Schmidt, C. E., and Leach, J. B. (2003). Neural tissue engineering: strategies for repair and regeneration. Annu. Rev. Biomed. Eng. 5, 293-347. doi: 10.1146/annurev.bioeng.5.011303.120731

Subramanian, A., Krishnan, U. M., and Sethuraman, S. (2009). Development of biomaterial scaffold for nerve tissue engineering: biomaterial mediated neural regeneration. J. Biomed. Sci. 16:108. doi: 10.1186/1423-0127-16-108

Sunyer, R., Jin, A. J., Nossal, R., and Sackett, D. L. (2012). Fabrication of hydrogels with steep stiffness gradients for studying cell mechanical response. PLoS ONE 7:e46107. doi: 10.1371/journal.pone.0046107

Tam, R. Y., Fuehrmann, T., Mitrousis, N., and Shoichet, M. S. (2014). Regenerative therapies for central nervous system diseases: a biomaterials approach. Neuropsychopharmacology 39, 169-188. doi: 10.1038/npp.2013.237

Teng, Y. D., Lavik, E. B., Qu, X., Park, K. I., Ourednik, J., Zurakowski, D., et al. (2002). Functional recovery following traumatic spinal cord injury mediated by a unique polymer scaffold seeded with neural stem cells. Proc. Natl. Acad. Sci. U.S.A. 99, 3024-3029. doi: 10.1073/pnas.052678899

Tian, L., Prabhakaran, M. P., and Ramakrishna, S. (2015). Strategies for regeneration of components of nervous system: scaffolds, cells and biomolecules. Regen. Biomater. 2, 31-45. doi: 10.1093/rb/rbu017

Ventre, M., Causa, F., and Netti, P. A. (2012). Determinants of cell-material crosstalk at the interface: towards engineering of cell instructive materials. $J$. R. Soc. Interface 9, 2017-2032. doi: 10.1098/rsif.2012.0308

Viventi, J., Kim, D. H., Moss, J. D., Kim, Y. S., Blanco, J. A., Annetta, N., et al. (2010). A conformal, bio-interfaced class of silicon electronics for mapping cardiac electrophysiology. Sci. Transl. Med. 2:24ra22. doi: 10.1126/scitranslmed.3000738

Viventi, J., Kim, D. H., Vigeland, L., Frechette, E. S., Blanco, J. A., Kim, Y. S., et al. (2011). Flexible, foldable, actively multiplexed, high-density electrode array for mapping brain activity in vivo. Nat. Neurosci. 14, 1599-1605. doi: $10.1038 / \mathrm{nn} .2973$
Wang, G. W., Yang, H., Wu, W. F., Zhang, P., and Wang, J. Y. (2017). Design and optimization of a biodegradable porous zein conduit using microtubes as a guide for rat sciatic nerve defect repair. Biomaterials 131, 145-159. doi: 10.1016/j.biomaterials.2017.03.038

Wang, H. B., Mullins, M. E., Cregg, J. M., Mccarthy, C. W., and Gilbert, R. J. (2010). Varying the diameter of aligned electrospun fibers alters neurite outgrowth and Schwann cell migration. Acta Biomater. 6, 2970-2978. doi: 10.1016/j.actbio.2010.02.020

WHO. (2006). "Neurological disorders: a public health approach," in Neurological Disorders: Public Health Challenges, eds J. A. Aarli, G. Avanzini, J. M. Bertolote, H. de Boer, H. Breivik, T. Dua, N. Graham, A. Janca, J. Kesselring, C. Mathers, A. Muscetta, L. Prilipko, B. Saraceno, S. Saxena, and T. J. Steiner (Geneva), 41-176.

Wong, A., Tang, Z., Park, I. H., Zhu, Y., Patel, S., Daley, G. Q., et al. (2011). Induced pluripotent stem cells for neural tissue engineering. Biomaterials 32, 5023-5032. doi: 10.1016/j.biomaterials.2011.03.070

Wylie, R. G., Ahsan, S., Aizawa, Y., Maxwell, K. L., Morshead, C. M., and Shoichet, M. S. (2011). Spatially controlled simultaneous patterning of multiple growth factors in three-dimensional hydrogels. Nat. Mater. 10, 799-806. doi: $10.1038 /$ nmat3101

Xie, J., Willerth, S. M., Li, X., Macewan, M. R., Rader, A. and SakiyamaElbert S. E. (2009). The differentiation of embryonic stem cells seeded on electrospun nanofibers into neural lineages. Biomaterials 30, 354-362. doi: 10.1016/j.biomaterials.2008.09.046

Yao, L., McCaig, C. D., and Zhao, M. (2009). Electrical signals polarize neuronal organelles, direct neuron migration, and orient cell division. Hippocampus 19, 855-868. doi: 10.1002/hipo.20569

Yao, L., Pandit, A., Yao, S., and McCaig, C. D. (2011). Electric field-guided neuron migration: a novel approach in neurogenesis. Tissue Eng. B. Rev. 17, 143-153. doi: 10.1089/ten.teb.2010.0561

Yao, L., Shanley, L., McCaig, C., and Zhao, M. (2008). Small applied electric fields guide migration of hippocampal neurons. J. Cell. Physiol. 216, 527-535. doi: $10.1002 /$ jcp. 21431

Yu, L. M. Y., Leipzig, N. D., and Shoichet, M. S. (2008). Promoting neuron adhesion and growth. Mater. Today 11, 36-43. doi: 10.1016/S1369-7021(08)70088-9

Zhang, Q. Y., Zhang, Y. Y., Xie, J., Li, C. X., Chen, W. Y., Liu, B. L., et al. (2014). Stiff substrates enhance cultured neuronal network activity. Sci. Rep. 4:6215. doi: $10.1038 /$ srep 06215

Conflict of Interest Statement: The authors declare that the manuscript was developed in the absence of any commercial or financial relationships that could be construed as a potential conflict of interest.

The handling Editor declared a shared affiliation, though no other collaboration, with the authors.

Copyright (C) 2018 Cangellaris and Gillette. This is an open-access article distributed under the terms of the Creative Commons Attribution License (CC BY). The use, distribution or reproduction in other forums is permitted, provided the original author(s) and the copyright owner are credited and that the original publication in this journal is cited, in accordance with accepted academic practice. No use, distribution or reproduction is permitted which does not comply with these terms. 